\title{
Microvascular exudative hyperresponsiveness in human coronavirus-induced common cold
}

Lennart Greiff, Morgan Andersson, Anders Åkerlund, Per Wollmer, Christer Svensson, Ulf Alkner, Carl G A Persson

\begin{abstract}
Background - The inflammatory response of the airway microcirculation in rhinitis and asthma may be recorded as luminal entry of plasma macromolecules (mucosal exudation). This study examines the exudative responsiveness of the subepithelial microvessels in subjects with and without common cold after inoculation with coronavirus.

Methods - The airway mucosa was exposed to exudative concentrations of histamine (40 and $400 \mu \mathrm{g} / \mathrm{ml}$ ) before and six days after inoculation. To assess whether mucosal penetration of a topically applied agent was altered, nasal absorption of chromium-51 labelled ethylene diamine tetraacetic acid $\left({ }^{51} \mathrm{Cr}\right.$ EDTA, MW 372) was also examined. A nasal pool technique kept the challenge and tracer solutes in contact with the same ipsilateral mucosal surface. Concentrations of albumin in lavage fluids were measured as an index of mucosal exudation of plasma. Nasal absorption of ${ }^{51} \mathrm{Cr}$-EDTA was determined by the cumulated 24 hour urinary excretion of radioactivity.
\end{abstract}

Results - Nine subjects developed common cold after coronavirus inoculation and 10 remained healthy. Histamine produced concentration dependent mucosal exudation of plasma in all subjects before and after coronavirus inoculation. In subjects with common cold, however, the histamine-induced mucosal exudation was significantly augmented compared with the group without common cold. This exudative hyperresponsiveness is not explained by an increased baseline exudation because the lavage regimen used produced comparably low baseline exudation in both groups of subjects, nor is it explained by an increased penetration of topical histamine because the ability of the nasal mucosa to absorb ${ }^{51} \mathrm{Cr}$-EDTA was not significantly increased in the subjects with common cold.

Conclusions - An increased proclivity of the airway subepithelial microcirculation to respond with plasma exudation develops during coronavirus-induced common cold. This specific exudative hyperresponsiveness may be a feature of inflammatory airway diseases.

(Thorax 1994;49:121-127)
Several reports have suggested that rhinoviral infections may contribute to the development of non-specific bronchial hyperresponsiveness. ${ }^{12}$ The possibility that viral infections of the nose also produce nasal hyperresponsiveness has not received attention. This seems unfortunate because nasal studies can be carried out with great specificity and yield information of potential relevance both for the upper and lower airways. ${ }^{3}$

Changes in the sensitivity and function of different nasal end organs including the epithelial barrier, the secretory apparatus, the innervation, and the subepithelial microcirculation can be assessed in the nose. ${ }^{3}$ One end organ response - the mucosal exudation of plasma - may be of special interest. This response constitutes a significant mucosal defence reaction ${ }^{4}$ but may also produce many pathogenetic effects in the airways. ${ }^{5}$ In addition, it can serve as a specific and quantitative index of the subepithelial inflammatory process. $^{3}$

In allergic airway disease and coronavirus infection the inflammatory process is characterised by mucosal exudation of bulk plasma. ${ }^{67}$ Topical challenge with single mediators such as histamine also produces a reproducible mucosal exudation of bulk plasma. ${ }^{89}$ Using histamine as a challenge agent we have recently shown that an exudative hyperresponsiveness may develop during seasonal allergic rhinitis. ${ }^{10}$ This increased vascular epithelial exudation response in allergy was not associated with increased mucosal absorption. ${ }^{11}$ It is not known whether the exudative responsiveness of the airway mucosa may be altered in other inflammatory respiratory diseases such as common cold. Viral infections are thought to damage the epithelium, resulting in increased tissue penetration of the challenge agent. This mechanism alone might produce exaggerated responses to airway challenges.

In the present study we have measured histamine-induced mucosal exudation of plasma before and during human coronavirusinduced common cold. We have also examined the rate of nasal absorption of 51-chromium labelled ethylene diamine tetraacetic acid $\left({ }^{51} \mathrm{Cr}-\mathrm{EDTA}, \mathrm{MW} 372\right)$ to assess whether the normal barrier function of the mucosa is altered in the present virus-induced inflammation. A nasal pool technique ${ }^{9}$ has been used both for histamine challenge and for administration of ${ }^{51} \mathrm{Cr}$-EDTA. This technique ensures that the challenge and tracer solutes, respectively, are kept in contact with a large and well defined area of the nasal mucosal surface for a selected period of time. 
Methods

SUBJECTS

Nineteen healthy men of mean age 24 (range 20-27 years) participated in the study. The subjects had no history of general, nasal, or allergic disease, and no history of recent drug treatment or recent vaccination. Absence of allergy was verified by a negative history and a negative skin prick test (Phazett, Pharmacia, Uppsala, Sweden). The study was performed in May 1991 and was approved by the local ethics committee and informed consent was obtained.

The subjects were isolated and lodged four by four in five separate two bedroom apartments. They were instructed not to meet people other than their lodge mates at a range closer than 10 metres. Outdoor activities were otherwise not restricted. All meals were delivered to the apartments. In order to ensure that the subjects were not infected at the start of the study, they were observed for the first two days of isolation. During this time none of the subjects developed symptoms of common cold so all were inoculated with coronavirus. Subjects not developing infection after coronavirus inoculation were used as control subjects.

NASAL CORONAVIRUS INOCULATION

Human coronavirus $229 \mathrm{E}$ was diluted in phosphate buffered saline at $4^{\circ} \mathrm{C}$ to a concentration of $100 \mathrm{TCID}_{50} / \mathrm{ml}$ (tissue culture infective dose 50 - that is, the dose that produced infection in $50 \%$ of cultures). With the subjects in a supine and extended neck position $0.5 \mathrm{ml}$ of this solution was administered into each nasal cavity two days after isolation (study day zero). In order to avoid infectious contamination during the study the investigators in contact with the subjects wore aprons, surgical gloves, and face masks. The non-disposable equipment used by the investigators was soaked in ethanol $(70 \%)$ or boiled water for two minutes between use. The clinical course, including daily measurements of symptoms and mucosal temperature, is reported elsewhere. ${ }^{?}$

\section{DETERMINATION OF COMMON COLD}

To distinguish between subjects with and without common cold a clinical evaluation was performed daily by an otolaryngologist. A symptom and clinical sign score, based on symptoms presented on direct questions and clinical signs on physical examination including an anterior rhinoscopy, was given twice daily according to the method of Beare $e t$ al. ${ }^{12}$ The criteria for the development of common cold was a total score of more than 16 points, and a convincing progress of symptoms and signs on the second to fifth days after inoculation - that is, a four point increase for at least two consecutive days. In support of the clinical evaluation an ELISA techique was used for serological analysis. Preinoculation sera were compared with convalescent sera obtained three to four weeks after inoculation. A greater than fourfold increase in the titre was con- sidered as seroconversion and as a suggestion of recent coronavirus-induced infection.

NASAL POOL TECHNIQUE

A nasal pool technique was used for concomitant histamine challenge and lavage, and for adminstration of the absorption tracer, respectively. ${ }^{9}$ The nasal pool device is a compressible plastic container equipped with a nasal adapter. The adapter is inserted into one of the nostrils (in the present study the right nostril) and the container is compressed by the subject sitting in a $60^{\circ}$ forward flexed neck position. The nasal pool fluid (in the present study $14 \mathrm{ml}$ ) is then instilled and maintained in contact with a large and defined area of the nasal mucosal surface for an extended period of time. When the pressure on the device is released the fluid returns into the container.

\section{MEASUREMENT OF MUCOSAL EXUDATION OF PLASMA}

Measurement of histamine-induced mucosal exudation of albumin was performed one month before and six days after coronavirus inoculation. Isotonic saline and histamine (40 and $400 \mu \mathrm{g} / \mathrm{ml}$ ) were introduced in sequence into the nasal cavity by the nasal pool technique. The fluid was maintained in the nasal cavity for 10 minutes, and 20 minutes elapsed between each instillation. To prevent histamine from being retained in the airway the mucosal surface was irrigated with saline for 30 seconds using the nasal pool technique, immediately after each 10 minute challenge. These lavage fluids were not collected. The recovered fluids were centrifuged $(105 \mathrm{~g}, 10$ minutes, $4^{\circ} \mathrm{C}$ ) and samples were obtained from the supernatant and frozen $\left(-20^{\circ} \mathrm{C}\right)$ while awaiting analysis. The concentration of albumin was measured in the recovered lavage fluids with a radioimmunoassay technique sensitive to $6.25 \mathrm{ng} / \mathrm{ml}$. The intra- and interassay coefficients of variation were $5 \%$ and $10 \%$, respectively.

Five days after inoculation the effect of repeated saline lavages without histamine on lavage fluid levels of albumin was examined in the group with common cold. Two consecutive saline lavages, each of 10 minutes duration, were performed, followed by a third lavage 30 minutes later. The concentration of albumin was measured in the recovered lavage fluids as described above.

\section{MEASUREMENT OF NASAL ABSORPTION OF} ${ }^{51}$ Cr-EDTA

Measurement of nasal absorption of ${ }^{51} \mathrm{Cr}$ EDTA was performed one month before and four days after coronavirus inoculation. ${ }^{13}{ }^{51} \mathrm{Cr}$ EDTA (about $5 \mathrm{MBq}$ ) in isotonic saline was introduced into the nasal cavity by the nasal pool technique. The fluid was maintained in the nasal cavity for 15 minutes. Two additional 30 second saline lavages were then promptly carried out to prevent absorption of any tracer retained on the mucosal surface. The nasal 
absorption of ${ }^{51} \mathrm{Cr}$-EDTA during the 15 minute exposure was determined by sampling the urine produced during 24 hours after the instillation procedure. Three samples (each $3.0 \mathrm{ml}$ ) taken from the pooled 24 hour urine collection were counted in a well counter for 2000 seconds. Two samples (each $3.0 \mathrm{ml}$ ) of a standard solution, prepared by diluting $0.1 \mathrm{ml}$ of the instillate to $100 \mathrm{ml}$, were counted together with the urine samples. The amount absorbed was expressed as the corresponding volume of the nasal instillate - that is, the urine count rate multiplied by the pooled urine volume, divided by the standard solution count rate corrected for dilution.

\section{STATISTICS}

The Friedman and Wilcoxon signed rank tests were used to examine differences in albumin concentrations between the three lavages performed on subjects with common cold five days after inoculation. The Wilcoxon signed rank test was used to examine differences in histamine-induced albumin concentrations and differences in absorbed volumes of ${ }^{51} \mathrm{Cr}$ EDTA before and after inoculation within the groups, with and without common cold. The Mann-Whitney $U$ test was used to examine differences in histamine-induced albumin concentrations between the groups with and without common cold. A regression analysis of individual values was used to examine the correlation between histamine-induced mucosal exudation of plasma on day six and absorption of ${ }^{51} \mathrm{Cr}$-EDTA on day four after inoculation. A p value $<0.05$ was considered significant. Data are presented as means (SE).

\section{Results}

Nine subjects developed common cold as judged by scores of symptoms and clinical signs (table), two of whom failed to show seroconversion. Because of a convincing development of symptoms, however, all nine subjects were included in the group with common cold. A total number of nine subjects showed seroconversion, two of whom were without convincing symptoms or clinical signs of common cold. The latter subjects were included in the group without common cold.

Before inoculation the albumin concentration in the saline lavage fluid obtained was low in subjects who subsequently developed or did not develop common cold $(11.8(3.5) \mu \mathrm{g} / \mathrm{ml}$ and $22 \cdot 3(5 \cdot 1) \mu \mathrm{g} / \mathrm{ml}$, respectively). Six days after inoculation the group without common cold also exhibited a relatively low albumin concentration $(34.6(8.5) \mu \mathrm{g} / \mathrm{ml})$ in the saline lavage fluid obtained, whereas the albumin concentration was significantly elevated (137.2 $(30.7) \mu \mathrm{g} / \mathrm{ml})$ in the group with common cold $(\mathrm{p}<0.01)$ reflecting an ongoing mucosal exudation of plasma. The initial saline lavage fluid obtained five days after inoculation (the day before the saline/histamine challenge series) in subjects developing common cold exhibited an almost equally elevated level of albumin concentration $(83.3(31.8) \mu \mathrm{g} / \mathrm{ml}$; $\mathrm{p}<0.05$ ), again demonstrating ongoing mucosal exudation in the subjects with common cold. However, in the second saline lavage fluid taken on day five a low baseline level of albumin $(34.1(11.2) \mu \mathrm{g} / \mathrm{ml})$ was obtained (Friedman test, $\mathrm{p}<0.05$; Wilcoxon's signed rank test, $p<0.01$ ) compared with the concentration of albumin in the first saline lavage fluid. Furthermore, in the third saline lavage fluid obtained 30 minutes later the level of albumin remained low $(36 \cdot 1(13.5) \mu \mathrm{g} / \mathrm{ml})$ suggesting that, with the present technique of repeated challenge and lavage experiments, changes in baseline concentrations of albumin would not invalidate the measurement of histamine-induced exudation in subjects who developed common cold.

Histamine produced a concentration dependent mucosal exudation of albumin in both groups before and six days after coronavirus inoculation (fig 1). Furthermore, the effect of histamine was increased in the group with common cold after inoculation compared with its effect before inoculation (histamine $40 \mu \mathrm{g} /$ $\mathrm{ml}(\mathrm{p}<0.05)$ and $400 \mu \mathrm{g} / \mathrm{ml}(\mathrm{p}<0.05))$. In contrast, the effect of histamine was unchanged in the group without common cold after inoculation compared with before inoculation. The

Individual scores of symptoms and clinical signs before inoculation and days 0-6 after inoculation, total scores after inoculation (days 1-6) according to method of Beare et al, ${ }^{12}$ and changes in coronavirus titre in subjects with and without common cold

\begin{tabular}{|c|c|c|c|c|c|c|c|c|c|c|c|}
\hline \multirow[t]{3}{*}{ Subject no. } & \multicolumn{9}{|l|}{ Symptom score } & \multirow[t]{3}{*}{ Increase in serotitre } & \multirow[t]{3}{*}{ Common cold } \\
\hline & \multirow{2}{*}{ Before inoculation } & \multicolumn{7}{|c|}{ Days after inoculation } & \multirow[t]{2}{*}{ Total } & & \\
\hline & & 0 & 1 & 2 & 3 & 4 & 5 & 6 & & & \\
\hline 1 & 0.0 & 0.0 & 1.0 & 2.5 & $11 \cdot 0$ & $23 \cdot 5$ & $12 \cdot 5$ & $5 \cdot 5$ & $56 \cdot 0$ & 0 & Yes \\
\hline 2 & 0.5 & 0.5 & $4 \cdot 5$ & 3.0 & 9.5 & $13 \cdot 5$ & $8 \cdot 5$ & 3.5 & 42.5 & $\geqslant 4$ fold & Yes \\
\hline 3 & 1.0 & 1.0 & 2.5 & 3.5 & $5 \cdot 5$ & $14 \cdot 0$ & $9 \cdot 5$ & $6 \cdot 0$ & $41 \cdot 0$ & $\geqslant 4$ fold & Yes \\
\hline 4 & 1.5 & 1.5 & $3 \cdot 5$ & $5 \cdot 0$ & $5 \cdot 0$ & 5.5 & $10 \cdot 0$ & $10 \cdot 0$ & $39 \cdot 0$ & 2 fold & Yes \\
\hline 5 & $0 \cdot 0$ & 1.5 & $2 \cdot 5$ & 1.5 & 6.5 & $11 \cdot 0$ & $8 \cdot 5$ & 3.5 & $33 \cdot 5$ & $\geqslant 4$ fold & Yes \\
\hline 6 & 1.5 & 1.5 & $2 \cdot 0$ & $3: 0$ & $7 \cdot 5$ & $11 \cdot 5$ & $3 \cdot 0$ & $5 \cdot 5$ & $32 \cdot 5$ & $\geqslant 4$ fold & Yes \\
\hline 7 & $0 \cdot 0$ & 0.0 & 0.0 & 1.0 & 1.5 & $5 \cdot 0$ & $12 \cdot 0$ & $7 \cdot 5$ & $27 \cdot 0$ & $\geqslant 4$ fold & Yes \\
\hline 8 & $2 \cdot 0$ & 1.5 & 1.5 & 2.5 & 5.0 & 5.5 & 5.5 & $3 \cdot 0$ & $23 \cdot 0$ & $\geqslant 4$ fold & Yes \\
\hline 9 & $0 \cdot 0$ & $0 \cdot 0$ & 0.0 & 1.0 & 2.5 & $4 \cdot 5$ & $8 \cdot 0$ & $5 \cdot 0$ & $21 \cdot 0$ & $\geqslant 4$ fold & Yes \\
\hline 10 & $2 \cdot 0$ & $2 \cdot 0$ & $3 \cdot 0$ & $6 \cdot 0$ & $6 \cdot 0$ & 3.0 & 4.5 & 5.5 & $28 \cdot 0$ & $\geqslant 4$ fold & No \\
\hline 11 & $1 \cdot 0$ & 1.0 & $4 \cdot 0$ & $3 \cdot 0$ & $4 \cdot 0$ & 4.5 & 3.5 & 3.5 & 22.5 & 0 & No \\
\hline 12 & 0.0 & 0.0 & 4.0 & $4 \cdot 0$ & 3.0 & $3 \cdot 0$ & $4 \cdot 0$ & $3 \cdot 0$ & $21 \cdot 0$ & 0 & No \\
\hline 13 & $0 \cdot 0$ & 0.0 & 1.0 & 2.5 & $2 \cdot 0$ & 5.5 & 3.5 & $4 \cdot 0$ & $18 \cdot 5$ & 0 & No \\
\hline 14 & 1.5 & $1 \cdot 0$ & $5 \cdot 0$ & $5 \cdot 5$ & $2 \cdot 5$ & 1.5 & 1.5 & 1.5 & 17.5 & 0 & No \\
\hline 15 & 0.0 & 2.5 & $2 \cdot 5$ & $2 \cdot 0$ & $3 \cdot 0$ & 2.5 & $3 \cdot 0$ & $2 \cdot 0$ & 15.0 & 0 & No \\
\hline 16 & 0.0 & $0 \cdot 0$ & $1 \cdot 0$ & 2.5 & 1.5 & $3 \cdot 0$ & $4 \cdot 0$ & $2 \cdot 0$ & $14 \cdot 0$ & $\geqslant 4$ fold & No \\
\hline 17 & $1 \cdot 0$ & 3.5 & $3 \cdot 0$ & $3 \cdot 0$ & $2 \cdot 0$ & 1.5 & 0.5 & 1.0 & $11 \cdot 0$ & 0 & No \\
\hline 18 & 0.0 & 1.0 & $3 \cdot 0$ & $2 \cdot 0$ & 0.0 & $0 \cdot 0$ & $2 \cdot 0$ & $2 \cdot 0$ & $9 \cdot 0$ & 0 & No \\
\hline 19 & 0.0 & $0 \cdot 0$ & 0.0 & 0.0 & $0 \cdot 0$ & $1 \cdot 0$ & 0.0 & 1.5 & 2.5 & 0 & No \\
\hline
\end{tabular}


histamine-induced levels of albumin were larger in the group with common cold than in the group without common cold (fig 1). The response to histamine was not different between the groups before inoculation.

If subjects with clinical signs of common cold but without seroconversion (subjects 1 and 4 in the table) and subjects without clinical signs of common cold but with seroconversion (subjects 10 and 16 in the table) are excluded from the calculations of histamine-induced mucosal exudation of albumin, the effect of histamine was still greater in the group with common cold than in the group without common cold (fig 2). The response to histamine was not different between the groups before inoculation.

Absorption of ${ }^{51} \mathrm{Cr}$-EDTA tended to increase in both the control and the common cold groups. However, the change was significant only in the control group. The absorbed volumes were $0.060(0.014) \mathrm{ml}$ before and $0.189(0.042) \mathrm{ml}$ after the inoculation in the group without common cold $(\mathrm{p}<0.05)$, and $0.091(0.035) \mathrm{ml}$ before and $0.194(0.053) \mathrm{ml}$ after inoculation in the group with common cold. Absorption of ${ }^{51} \mathrm{Cr}$-EDTA was similar for subjects with and without common cold before and after inoculation. There were no
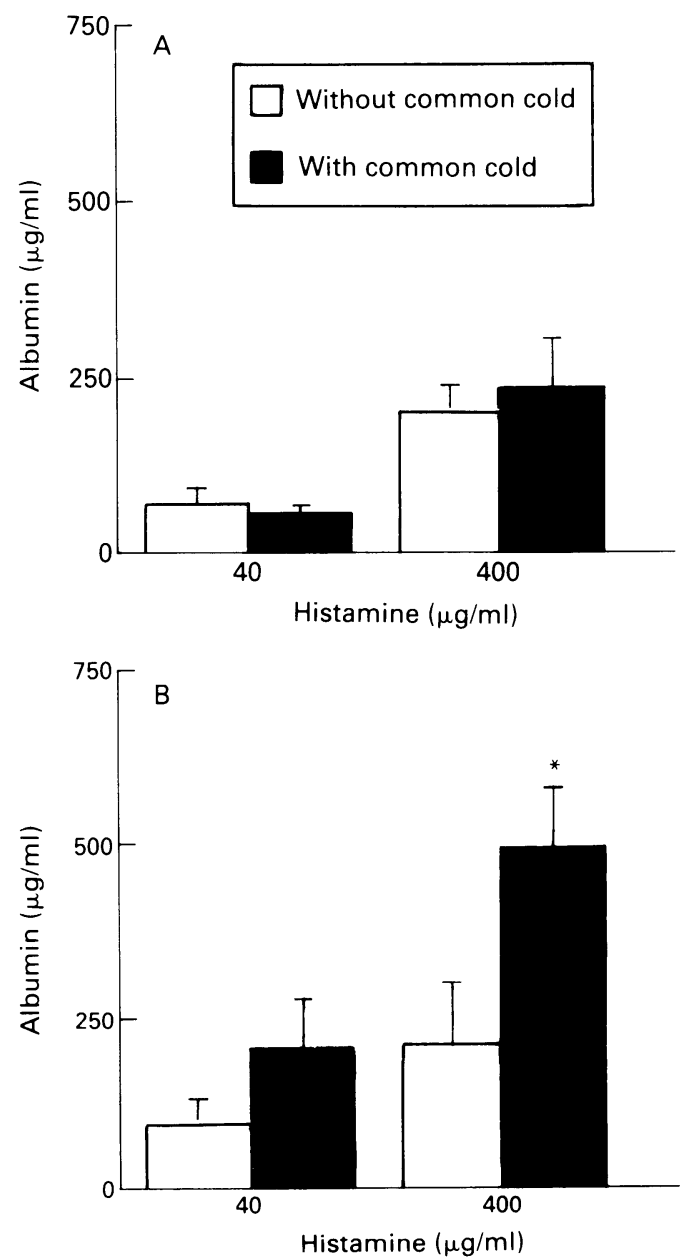

Figure 1 Histamine-induced mucosal exudation of albumin $(A)$ before and $(B)$ six days after coronavirus inoculation in groups with ( $n=9$ ) and without ( $n=10$ ) common cold. After coronavirus inoculation an

exudative hyperresponsiveness was seen in the group with common cold compared with group without common cold $(* p<0.05)$. significant correlations between histamineinduced exudation of albumin and absorption of ${ }^{51} \mathrm{Cr}$-EDTA after inoculation at any histamine concentration and in any of the groups $(r=0 \cdot 16-0 \cdot 47, \mathrm{p}>0 \cdot 05)$.

\section{Discussion}

As previously reported ${ }^{7}$ the symptoms and signs of common cold of the present subjects reached a maximum after four to five days and were accompanied by mucosal exudation of bulk plasma reflecting the coronavirusinduced airway inflammation. This study showed an increased exudative responsiveness to topical histamine in the subjects with coronavirus-induced common cold, and also found that the nasal absorption of ${ }^{51} \mathrm{Cr}$-EDTA was not significantly increased in these subjects.

In this study histamine produced concentration dependent mucosal exudation of plasma in all subjects before and after inoculation. We have demonstrated previously by analyses of lavage fluid levels of albumin, fibrinogen, and $x_{2}$-macroglobulin that the histamine-induced
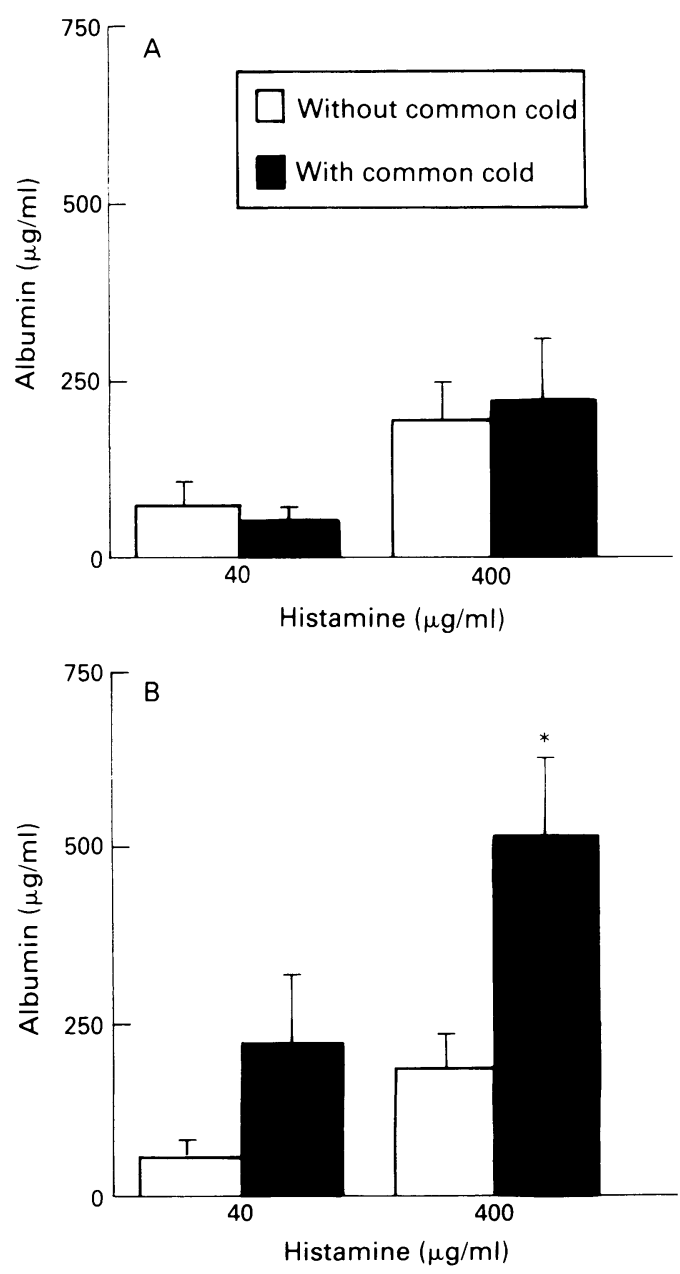

Figure 2 Histamine-induced mucosal exudation of albumin $(A)$ before and ( $B$ ) six days after coronavirus inoculation in groups with $(n=7)$ and without $(n=8)$ common cold. Subjects 1, 4,10, and 16 are excluded either because they had symptoms and signs of common cold without seroconversion or they had no symptoms and signs of common cold but showed seroconversion (see table). After coronavirus inoculation an exudative hyperresponsiveness was seen in the group with common cold compared with the group without common cold cold compared 
exudation of albumin corresponds to mucosal exudation of non-sieved bulk plasma. ${ }^{310} \mathrm{We}$ have also observed, in human nasal airways ${ }^{13}$ and in guinea pig tracheobronchial airways, ${ }^{14}$ that histamine-induced mucosal exudation of plasma is not associated with any change in the absorption permeability of the airway mucosa.

Interestingly, the histamine-induced exudation of plasma was significantly greater in subjects with common cold than in those without common cold. The response in the former group was significant. For comparison, about a fivefold greater concentration of histamine may be required to produce the same degree of plasma exudation in healthy subjects. ${ }^{9}$ The increased response in subjects with common cold may not reflect a high baseline concentration of albumin because control experiments carried out five days after inoculation showed that the high baseline is already lost after the initial saline lavage. Furthermore, the results obtained on day five showed that albumin does not accumulate significantly on the mucosal surface for the period of 30 minutes that was chosen in the present challenge and lavage regimen. The baseline levels obtained on day five thus strongly suggest that the abnormally increased concentrations of albumin after the two histamine challenge doses in subjects with common cold reflect an exudative hyperresponsiveness of the airway mucosa. This hyperresponsiveness is similar in magnitude to that recently found in seasonal allergic rhinitis. ${ }^{10}$ Hence, both allergen-induced and virusinduced airway inflammation appear to be associated with exudative hyperresponsiveness. We have suggested that exudative indices may directly reflect the intensity of the subepithelial airway inflammation. ${ }^{3}$ One consequence of an increased exudative responsiveness, as observed in the present study, is that the mucosal exudation response would be an even more sensitive measure of airway inflammation than previously suggested. ${ }^{3}$

In a series of studies involving human and guinea pig airways we have examined mechanisms of mucosal exudation of plasma. ${ }^{315}{ }^{16}$ Positioned just beneath the epithelial lining is a network of capillary venular microvessels. Active separation of endothelial cells of postcapillary venules is induced by actions of inflammatory mediators on these cells. Through the open gaps in the venular wall bulk plasma is extravasated along a hydrostatic pressure gradient. We have suggested that the extravasated plasma moves up between epithelial cells and exerts a hydrostatic pressure load on basolateral aspects of the cells. ${ }^{315}$ This load may produce a transient separation of the junctions between the epithelial cells so that a paracellular pathway for the clearance of plasma into the lumen is created. In guinea pig tracheal tube preparations we have shown that an increase in serosal hydrostatic pressure of about $5 \mathrm{~cm} \mathrm{H}_{2} \mathrm{O}$ is sufficient to move macromolecules from the serosal to the mucosal aspect of the preparation. ${ }^{16}$ Furthermore, the luminal entry in vitro is not affected by the presence of exudative mediators on the mucosa, ${ }^{16}$ suggesting that the physical pressure effect alone, without the aid of any mediator-induced epithelial effect, accounts for the passage. Luminal entry of bulk plasma may thus occur along a small hydrostatic pressure gradient, and even if mucosal exudation of plasma is pronounced it may occur without generation of oedema ${ }^{17}$ or increased lymph protein transport. ${ }^{18}$ Mucosal exudation of plasma is produced by threshold inflammatory challenges. ${ }^{19}$ Hence, the movement of extravasated plasma into the airway lumen may not be hindered greatly by the normal epithelial lining and may not involve pharmacological effects on the epithelial cells. We therefore suggest that the increased exudative responsiveness to histamine challenge in coronavirus-induced airway inflammation does not reflect an abnormal responsiveness of the epithelial lining, but is the result of an increased responsiveness of the subepithelial microcirculation.

Mucosal exudation of plasma is strictly unidirectional and the baseline absorption ability of the mucosa is not affected by the exudation process..$^{131420}$ In the present study nasal absorption of ${ }^{51} \mathrm{Cr}$-EDTA was not significantly changed in subjects with common cold at the height of infection, suggesting that significant epithelial destruction had not occurred. Since mucosal exudation of plasma may occur without damage to the epithelial lining, this process may be considered a first line respiratory defence also in common cold.

Mucosal exudation of plasma is induced by inflammatory mediators, ${ }^{819}$ allergen, ${ }^{61921}$ occupational factors, ${ }^{19}$ and infection. ${ }^{7}$ The intensity and time course of the plasma exudation response can be monitored by analysing the concentration of plasma proteins/tracers in airway mucosal surface liquids. Mucosal exudation of bulk plasma has been found in allergic rhinitis ${ }^{21}$ and in allergic asthma. ${ }^{6}$ Furthermore, increased concentrations of both albumin and fibrinogen have been shown in airway mucosal surface liquids after nasal coronavirus inoculation, indicating that mucosal exudation of bulk plasma is ongoing in subjects with common cold, ${ }^{722}$ particularly late at night and in the early morning hours. ${ }^{22}$ In the present study the first saline lavages carried out on days five and six in subjects with common cold revealed abnormally high levels of albumin, confirming the inflammatory exudative nature of this disease.

The mechanism of the exudative hyperresponsiveness has not yet been clarified. In the present study the nasal absorption of ${ }^{51} \mathrm{Cr}$ EDTA (a small polar solute like histamine) was not significantly changed in subjects with common cold. It therefore seems that increased penetration of histamine to the subepithelial microvascular target can be excluded as an important mechanism. Although it has been suggested that epithelial disruption and increased absorption across the mucosa is a feature of airway inflammation and airway hyperresponsiveness, ${ }^{23-25}$ this is not strongly supported by actual observations made in asthmatic subjects, ${ }^{2627}$ and in experimental studies involving human nasal airways and animal tracheobronchial airways. ${ }^{131420}$ We 
have shown that absorption across the nasal mucosa may even be reduced in seasonal allergic rhinitis late into the birch pollen season. ${ }^{11}$ During the course of the present study the absorption rate was slightly increased both in healthy individuals and in those with common cold. This effect may be a result of the frequent lavages that were carried out in these subjects during the course of the entire experiment comprising seven days. ${ }^{7}$ It is less likely that coronavirus inoculation in itself may have contributed to the increased absorption that was recorded in subjects who did not develop common cold.

Bronchial hyperresponsiveness to topical histamine has been shown during virus infection, ${ }^{1}$ but it is not known what mechanisms are involved in this response. Indeed, if the mucosal thickness is increased this could in theory explain an increased "obstructive" responsiveness to any fixed bronchoconstrictor stimulus. Thus, methacholine and histamine have previously been employed to study non-specific responsiveness in asthma (and rhinitis). In the present study we have used histamine specifically to examine the ability of the subepithelial microcirculation to respond with plasma exudation. The presently observed increased mucosal responsiveness may be one of several specific end organ functions of the airways that can potentially be altered in airway inflammation. Other important functions include sensory nerve responsiveness and secretory responsiveness, both of which may increase in airway diseases. ${ }^{3}$

It is difficult to examine plasma exudation and solute absorption across the human tracheobronchial mucosa in vivo with acceptable specificity. The distribution of absorption tracers and challenge factors may not be well controlled, and it may be difficult to distinguish between airway and alveolar absorption processes in the human lung. In a series of studies on human nasal and guinea pig tracheobronchial airways we have found very similar responses to inflammatory challenges between the upper and the lower airways, particularly concerning effects on plasma exudation and solute absorption rates. ${ }^{9131428}$ The possibility that an exudative hyperresponsiveness may also be present in inflammation of the lower airways cannot therefore be excluded. Studies on exudative responsiveness may be particularly warranted in asthma where exuded plasma is considered a multipotential disease mechanism. ${ }^{5}$

It is possible that altered airway end organ functions may be important and specific characteristics of inflammatory airway diseases or of subgroups of patients within a particular disease. Our observation of increased exudative responsiveness in both virus-induced (this study) and allergen-induced ${ }^{10}$ inflammation suggests a generality of this particular alteration in mucosal function. However, we do not yet know any details of the development of increased exudative responsiveness or its role in the disease process. It is an intriguing, but as yet unproven, possibility that this specific responsiveness may be modulated by antiinflammatory drug intervention.

We conclude that coronavirus-induced common cold is characterised by an increased proclivity of the subepithelial microcirculation to respond with plasma exudation. This exudative hyperresponsiveness may not be explained by increased mucosal penetration of histamine, as the absorption permeability of the airway was not significantly changed in the subjects with common cold.

This study was supported by the Swedish Medical Research Council (Projects 8308 and 2872), the Medical Faculty of Lund University, the Swedish Association against Asthma and Allergy, and Skaraborg County Council.

1 Empey DW, Laitinen LA, Jacobs L, Gold WM, Nadel JA. Mechanisms of bronchial hyperreactivity in normal subjects after respiratory tract infection. Am Rev Respir Dis 1976;113:131-9.

2 Bardin PG, Johnston SL, Pattemore PK. Viruses as precipitants of asthma symptoms. II. Physiology and mechanisms. Clin Exp Allergy 1992;22:809-22.

3 Persson CGA, Svensson C, Greiff L, Anderson $M$, Wollmer P, Alkner U, et al. Use of the nose to study the inflammatory response in the respiratory tract. Thorax 1992;47:993-1000.

4 Persson CGA, Erjefält I, Alkner U, Baumgarten C, Greiff $\mathrm{L}$, Gustafsson B, et al. Plasma exudation as a first line respiratory mucosal defence. Clin Exp Allergy respiratory muc

5 Persson CGA. Role of plasma exudation in asthmatic airways. Lancet 1986;ii:1126-9.

6 Salomonsson P, Grönneberg R, Gilljam H, Andersson O, Billing B, Enander I, et al. Bronchial exudation of bulk plasma at allergen challenge in allergic asthma. $A m$ Rev Respir Dis 1992;146:1535-42.

7 Akerlund A, Greiff L, Andersson M, Bende M, Alkner U, Persson CGA. Mucosal exudation of fibrinogen in coronavirus-induced common cold. Acta Otolaryngol (Stockh) 1993;113:642-8.

8 Svensson C, Baumgarten CR, Pipkorn U, Alkner U, Persson CGA. Reversibility and reproducibility of histamine induced plasma leakage in nasal airways. Thorax 1989;44:13-8.

9 Greiff L, Pipkorn U, Alkner U, Persson CGA. The "nasal pool-device" applies controlled concentrations of solutes on human nasal airway mucosa and samples its surface exudation/secretions. Clin Exp Allergy 1990;20:253-9.

10 Persson CGA, Greiff L, Svensson C, Andersson M, Åkerlund A, Wollmer P, et al. Exudative hyperresponsiveness of the airway microcirculation in allergic rhinitis and common cold (abstract). Am Rev Respir Dis 1993; 147:A833.

11 Greiff L, Wollmer P, Svensson C, Andersson M, Persson CGA. Effects of seasonal allergic rhinitis on airway mucosal absorption of chromium-51 labelled EDTA. Thorax 1993;48:648-50.

12 Beare A, Reed S. The study of anti-viral compounds in volunteers. In: Oxford JS, ed. Chemoprophylaxis and virus infections of the respiratory tract. Vol 2. Cleveland: CRC Press, 1977:28-55.

13 Greiff L, Wollmer P, Pipkorn U, Persson CGA. Absorption of ${ }^{51} \mathrm{Cr}$-EDTA across the human nasal mucosa in the of ${ }^{21}$ Cr-EDTA across the human nasal mucosa in the
presence of topical histamine. Thorax 1991;46:630-2.

14 Greiff L, Erjefält I, Wollmer P, Pipkorn U, Persson CGA. Effects of histamine, ethanol, and a detergent on exudation and absorption across the guinea pig airway mucosa in vivo. Thorax 1991;46:700-5.

15 Persson CGA. Permeability changes in obstructive airway disease. In: Sluiter HJ, Van der Lende R, eds. Bronchitis IV. Assen: Van Gorcum, 1989:236-46.

16 Persson CGA, Erjefält I, Gustafsson B, Luts A. Subepithelial hydrostatic pressure may regulate plasma exudation across the mucosa. Int Arch Allergy Appl Immunol 1990;92:148-53.

17 Erjefält I. Plasma exudation in tracheobronchial airways: a physiological and pharmacological study in the guinea pig. Thesis, Lund University, Sweden: 1991.

18 Erjefält I, Luts A, Persson CGA. The appearance of airway exudation and absorption tracers in guinea pig tracheobronchial lymph nodes. $\mathcal{f}$ Appl Physiol 1993;74:817-24.

19 Erjefält I, Persson CGA. Inflammatory passage of plasma macromolecules into airway wall and lumen. Pulmonol Pharmacol 1989;2:93-102.

20 Erjefält I, Persson CGA. Allergen, bradykinin, and capsaicin increase outward but not inward macromolecular permeability of guinea-pig tracheobronchial mucosa. Clin Exp Allergy 1991;21:217-24.

21 Svensson C, Klementsson H, Alkner U, Pipkorn U, Persson CGA. A topical glucocorticoid reduces the level of fibrinogen and bradykinins on the allergic nasal mucosa during natural pollen exposure. Allergy 1993 (in press).

22 Persson CGA, Greiff L, Ákerlund A, Andersson M, Svensson $\mathrm{C}$, Alkner $\mathrm{U}$. The mucosal inflammatory process is (acturnal Am Rev Respir Dis 1993;147:A66. 
23 Buckle FG, Cohen AB. Nasal mucosal hyperpermeability to macromolecules in atopic rhinitis and extrinsic asthma. $\mathcal{f}$ Allergy Clin Immunol 1975;55:213-21.

24 Hogg JC, Eggleston PA. Is asthma an epithelial disease? $A m$ Rev Respir Dis 1984;129:207-8.

25 Laitinen LA, Heino M, Laitinen A, Kava T, Haahtela T. Damage of the airway epithelium and bronchial reactivity in patients with asthma. Am Rev Respir Dis 1985;131:599606.

26 Elwood RK, Kennedy S, Belzberg A, Hogg JC, Pare PD.
Respiratory mucosal permeability in asthma. Am Rev Respir Dis 1983;128:523-7.

27 O'Byrne PM, Dolovich M, Dirks R, Roberts RS Newhouse MT. Lung epithelial permeability: relation to non-specific airway responsiveness. F Appl Physiol 1984;57:77-84.

28 Greiff L, Wollmer P, Erjefailt I, Pipkorn U, Persson CGA. Clearance of $99 \mathrm{~m}$ Tc-DTPA from guinea pig nasal tracheobronchial, and bronchoalveolar airways. Thorax 1990;45:841-5. 